\title{
UNIVERSAL QUATERNARY QUADRATIC FORMS
}

D. C. MORROW

Let the positive quaternary quadratic form $F=\sum_{i, j=1}^{4} b_{i j} x_{i} x_{j}$, where $b_{i j}$ are integers and $b_{i j}=b_{j i}$, have its hessian equal to $H^{\prime}$. Then it can be shown that every positive quaternary quadratic form whose hessian is $H^{\prime}$ is equivalent to a form $F$ in which $b_{11} \leqq\left(4 H^{\prime}\right)^{1 / 4}, 2\left|b_{i j}\right|$ $\leqq b_{11}, j=2,3,4$, and $b_{11} F-\left(\sum_{j=1}^{4} b_{1 j} x_{j}\right)^{2}$ is a reduced positive ternary quadratic form $T=\sum_{i, j=2}^{4} B_{i j} x_{i} x_{j}$ whose hessian is $b_{11}^{2} H^{\prime}$. Further if $b_{11}=2$, we may take $b_{1 j} \geqq 0, B_{23} \geqq 0$ and $B_{22} B_{34}-B_{23} B_{24} \geqq 0$, and in case $B_{23}=0$ we may choose $B_{24} \geqq 0$. A form satisfying these conditions is a reduced form.

This paper is concerned with the determination of the positive forms $f_{0}=\sum_{i, j=1}^{4} c_{i j} x_{i} x_{j}$, in which $c_{i j}=c_{j i}, c_{i i}$ and $2 c_{i j}$ are integers, which are universal. For the case in which $c_{i j}=0$ if $i \neq j$ the forms which represent all positive integers have been determined by Ramanujan. ${ }^{1}$ L. E. Dickson ${ }^{2}$ considered those forms in which $c_{1 j}=0$, $j=2,3,4$. Since the minimum positive integer which is represented by $f_{0}$ is 1 , this form is equivalent to a form $f$ whose first coefficient is 1. Let $F=2 f=\sum_{i, j=1}^{4} a_{i j} x_{i} x_{j}$, where $a_{i j}=a_{j i}$ are integers, $a_{11}=2$ and $a_{i i} \equiv 0(\bmod 2)$, be a reduced form whose hessian is $H$. Then $4 f=X^{2}$ $+T$ where $X=\sum_{j=1}^{4} a_{1 j} x_{j}$ and $T=\sum_{i, j=2}^{4} A_{i j} x_{i} x_{j}\left(A_{i j}=a_{11} a_{i j}-a_{1 i} a_{1 j}\right)$ is a reduced positive ternary quadratic form whose hessian is $4 H$. Since $f$ is universal, $4 f$ represents all positive multiples of 4 . The minimum positive integer $M$ which is represented by the ternary $T$ is $\leqq 8$ and $\equiv 0$ or $3(\bmod 4)$. Hence $M=3,4,7$ or 8 .

The ternary form ${ }^{3} M T=U^{2}+Q$ where $U=M x_{2}+A_{23} x_{3}+A_{24} x_{4}$, and $Q=A x_{3}^{2}+2 B x_{3} x_{4}+C x_{4}^{2}$ is a reduced binary quadratic form whose hessian is $4 M H$, and where $A=M A_{33}-A_{23}^{2}, B=M A_{34}-A_{23} A_{24}$ and $C=M A_{44}-A_{24}^{2}$, while $2\left|A_{23}\right|$ and $2\left|A_{24}\right|$ are each $\leqq M$. We may choose $A_{23} \geqq 0$ and $B \geqq 0$, while if $A_{23}=0$, we may take $A_{24} \geqq 0$.

The least positive integer $A$ which is represented by $Q$ if $M=3$ is $\leqq 24$ and $\equiv 0$ or $8(\bmod 24)$. If $M=4, A \leqq 48$ and $\equiv 0$ or $12(\bmod 16)$ if $A_{23}=0$, and $A \equiv 12$ or $8(\bmod 16)$ if $A_{23}=2$. For $M=7, A \leqq 84$ and $\equiv 0,-4,-8$, or $-16(\bmod 28)$ according as $A_{23}=0,1,2$ or 3 . Finally

Presented to the Society, April 6,1928, under the title All quaternary quadratic forms which represent every positive integer; received by the editors November 26, 1946, and, in revised form, November 9, 1947.

1 Proc. Cambridge Philos. Soc. vol. 19 (1916-1919) pp. 11-21.

2 Amer. J. Math. vol. 49 (1927) pp. 39-56.

' Landau, Handbuch ... Verteilung der Primzahlen, 1909, p. 545. 
if $M=8, A \leqq 160$ and $A \equiv 0,8,16,20,24$ or $28(\bmod 32)$. It follows that the number of reduced universal forms is finite.

The problem has not been completely solved, since there are a number of forms for which, as yet, only empirical results have been obtained. The methods used are, largely, those used by L. E. Dickson. The results have been put in tabular form and are being sent to Professor Dresden for deposit in the library of the Society. Table I gives those forms, 1417 in number, which have been proved to be universal. Since the definition given for a reduced form does not always yield a unique reduced form for each class, certain of the forms in this table will be equivalent. In table II are listed those forms which satisfy the necessary conditions for universality, but which fail to represent certain integers. These integers are specified in the table.

WAYNE UNIVERSITY 